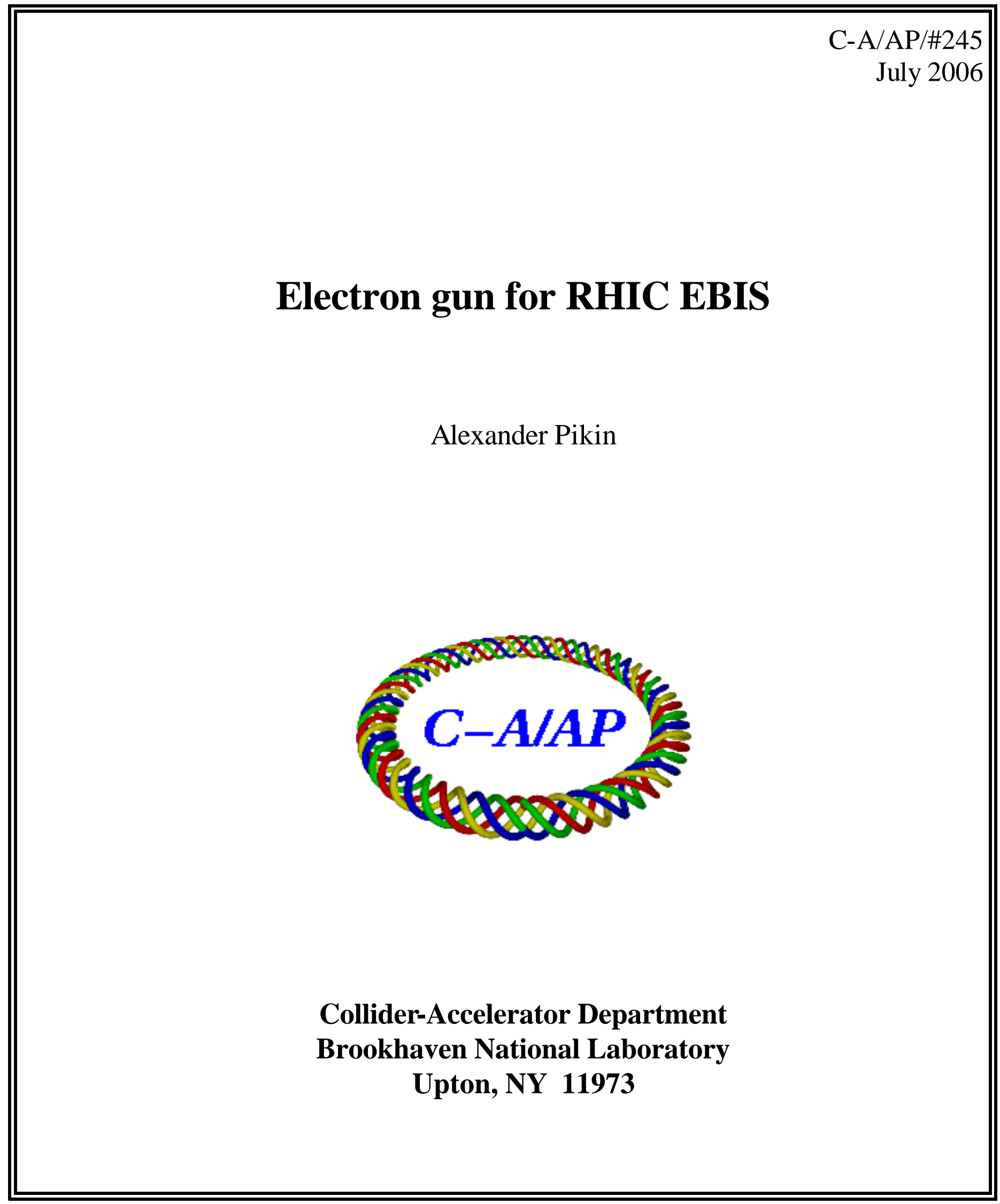




\title{
Electron gun for RHIC EBIS
}

\author{
Alexander Pikin
}

\section{Introduction}

The method of forming the electron beam in a new gun for RHIC EBIS is the same as is being used for a Test EBIS gun: purely magnetic focusing of the electron beam with cathode positioned in a magnetic field sufficient for acceptable amplitude of radial beam oscillations. The prototype electron gun was simulated and designed by a Novosibirsk team $[1,2]$ and demonstrated exceptional performance in generating the electron beam with designed parame ters, stable and flexible for control, capable for deep deceleration in a drift tube region and in the electron collector.

\section{Features of the electron gun}

- RHIC requirements for the ion beam intensity presume use of electron beam current in EBIS up to $\mathrm{I}_{\mathrm{e} l}=20 \mathrm{~A}$, which is twice higher than the existing electron beam current. The existing electron gun will not be able to meet this requirement without modifications. It would be preferable to have a gun with perveance higher than the existing gun $\left(1.4 \times 10^{-6} \mathrm{~A} / \mathrm{V}^{3 / 2}\right)$ to operate (ideally) with two TREK-20/20 anode power supplies for generation of a $20 \mathrm{~A}$ electron beam.

- The power deposition of electron beam on an electron collector has maximum on an axial power density distribution corresponding to the peripheral part of the electron beam. It is desirable to reduce this peak by depleting emission from the peripheral part of the cathode.

- Operation of EBIS on the acceleration facility presume a fast turn-around time for any repair works including replacement of the electron gun, which should be provided by the gun design and the technology of such replacement.

\section{Design of the electron gun}

The design of the new electron gun is based on the existing Test EBIS gun. The modifications include:

- Assembly of the cathode and anode in a single rigid unit, which allows aligning these electrodes on a bench before mounting them inside a gun vacuum chamber, not on different parts of the gun chamber it is in an existing prototype. The 
electron gun assembly with a prototype configuration of electrodes is presented in Fig 1. The gun has sufficient electrical insulation to generate a 20 A electron beam with low perveance $\left(1.4 \times 10^{-6} \mathrm{~A} / \mathrm{V}^{3 / 2}\right)$ if needed.

- The basic perveance of the new electron gun is approximately twice higher than has the existing prototype, which allows using lower anode voltage to generate the same electron currents (Fig. 2). The design allows control of the perveance of the electron gun by changing the cathode-anode distance using spacers with different lengths.

- The cathode-anode assembly will be suspended on a single isolated water-cooled copper rod (Fig. 3), not on two tubes capable of producing a tilt of the gun axis as a result of their thermal expansion as in an existing gun. The new design is expected to preserve the position and orientation of the gun axis during all regimes of the gun operation.

- The Wehnelt electrode in a new gun is isolated from the cathode. By applying different voltages on the Wehnelt electrode with respect to the cathode it is possible to control the electron emission from the periphery of the cathode, shaping the radial profile of the electron beam similar to Fermilab gun [4]. The basic Wehnelt electrode extends over the cathode and partially shields its periphery (Fig. 4).

- As an option a Wehnelt electrode with existing (not shielded geometry, Fig. 5) is designed and can be used as an alternative to the shielded geometry of the Wehnelt electrode.

- The gun in operation can be replaced with an identical second unit without venting either of them to atmosphere. The gun chamber is separated from the rest of EBIS with two gate valves and a buffer chamber between them (Fig. 6). In case if there is a need to replace the gun (cathode failure, isolation problem, etc.) both gate valves will be closed, the buffer chamber vented, the gun chamber with its gate valve disconnected from the buffer chamber and removed. Another gun chamber with its gate valve will be installed in place of the first one and sealed. Buffer chamber will be pumped out and after an acceptable vacuum is achieved the both gate valve will be opened and gun volume will be connected to the volume of the rest of the EBIS. No venting of the gun chamber or EBIS is necessary in this method and operation after the gun replacement is hoped to be within several hours. 


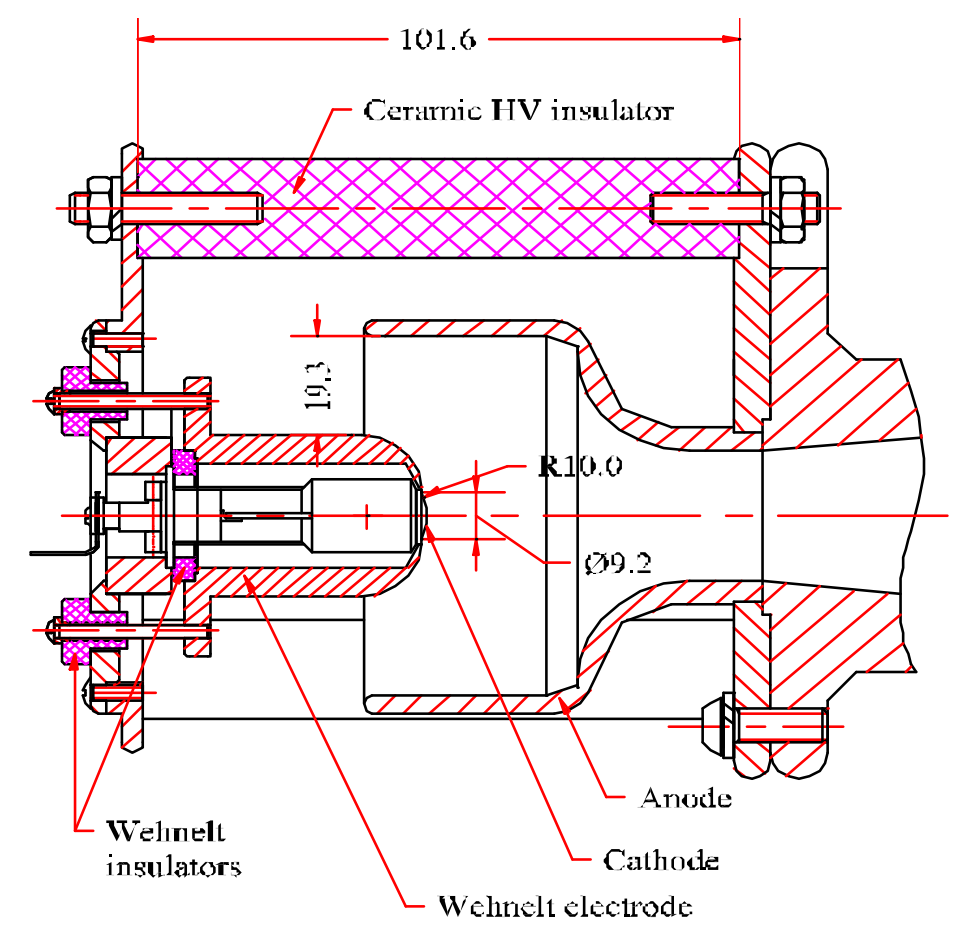

Fig. 1. Low perveance electron gun (prototype geometry).

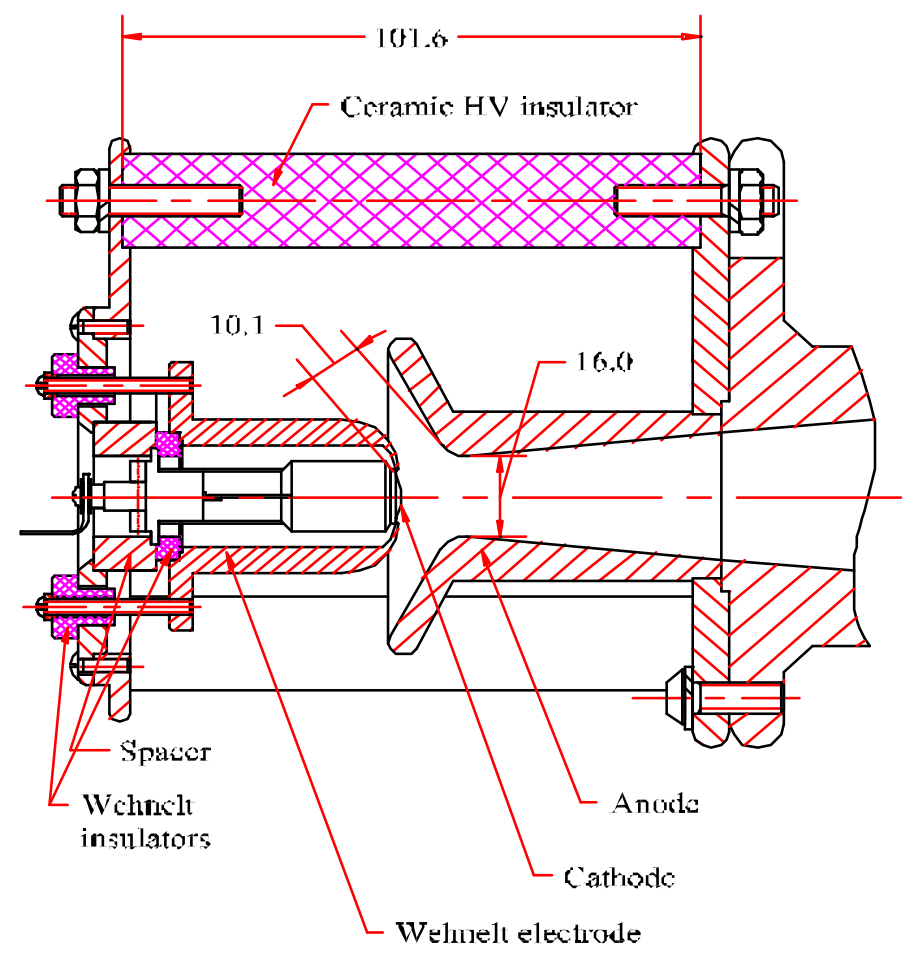

Fig. 2. High perveance Electron gun. 


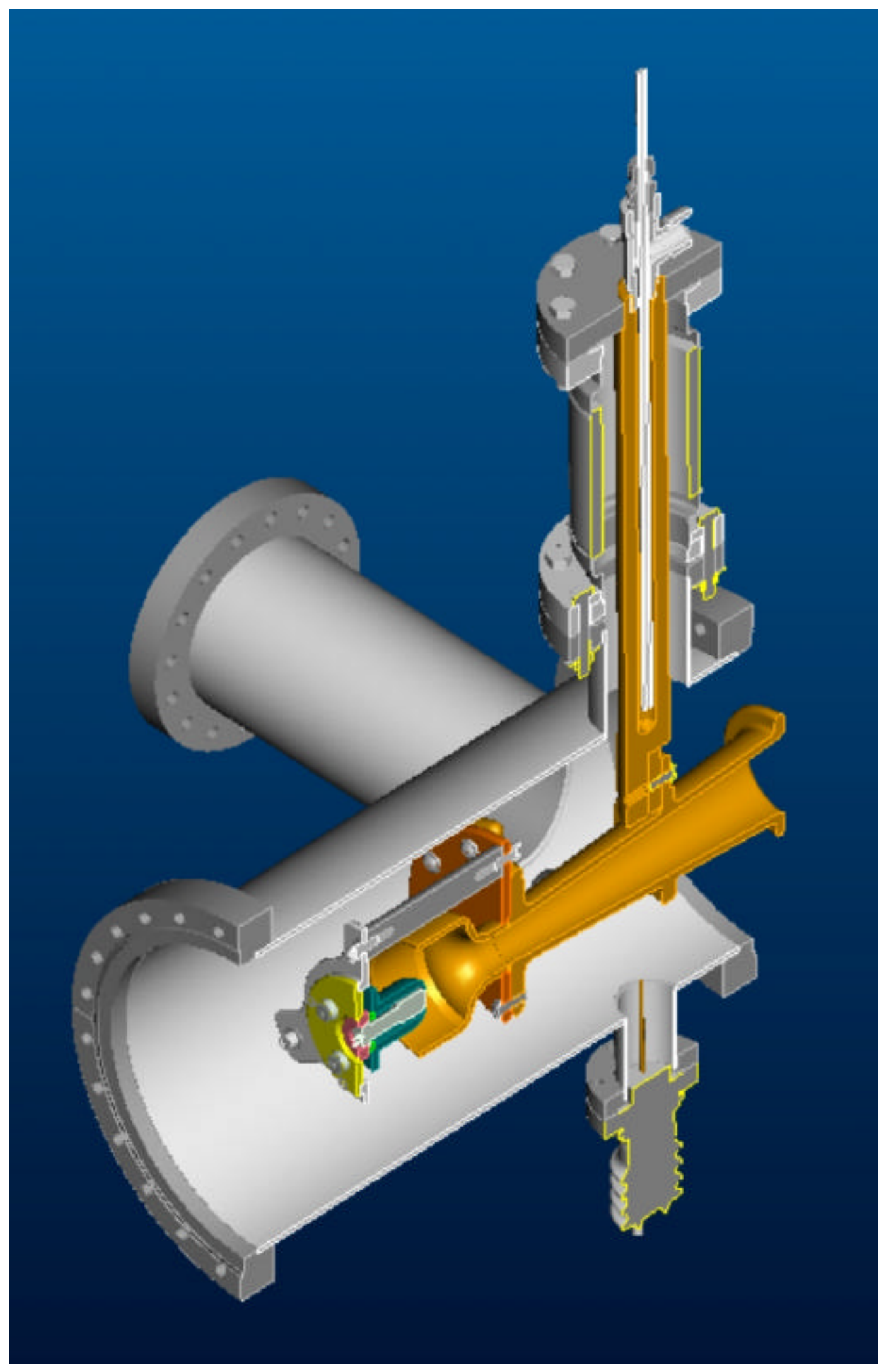

Fig. 3. Electron gun assembly (low perveance version) in a vacuum chamber. 


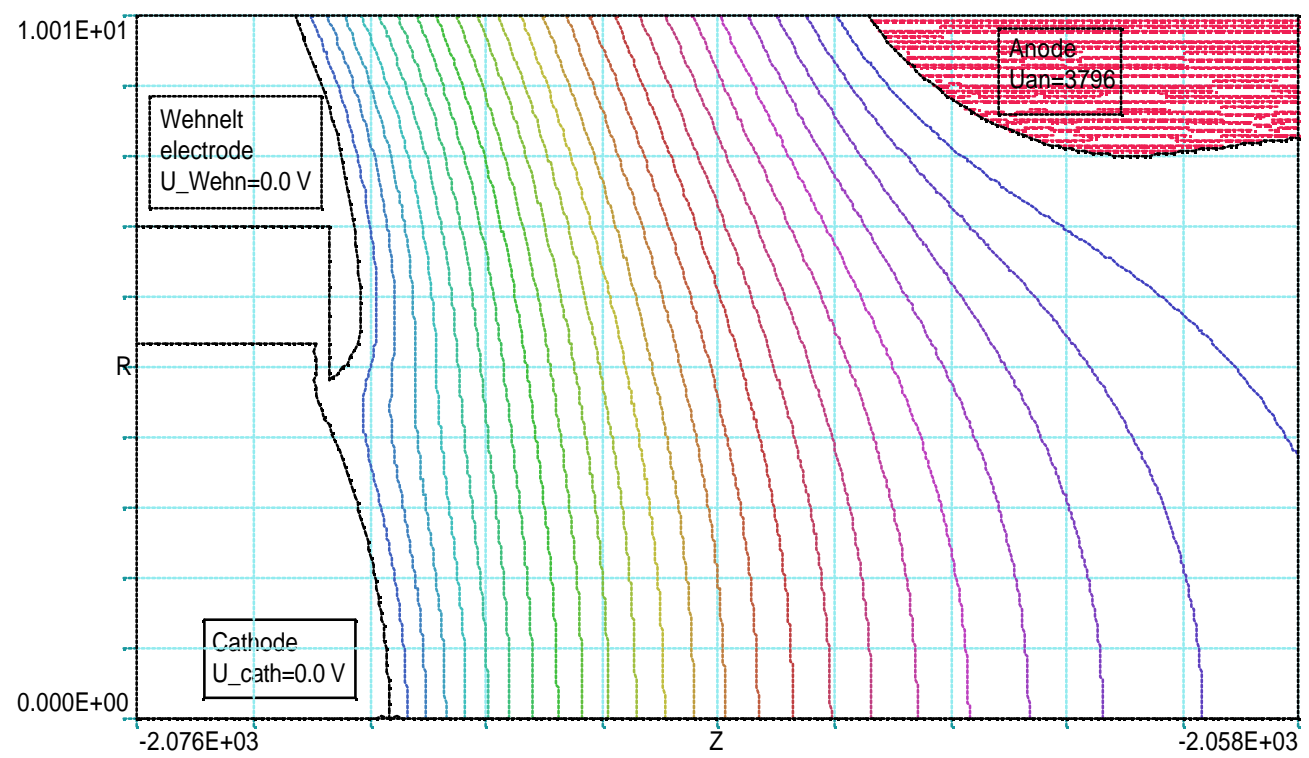

Fig. 4. Electrodes geometry of the high perveance electron gun with shielded Wehnelt electrode and electric field distribution with zero bias of Wehnelt with respect to the cathode.

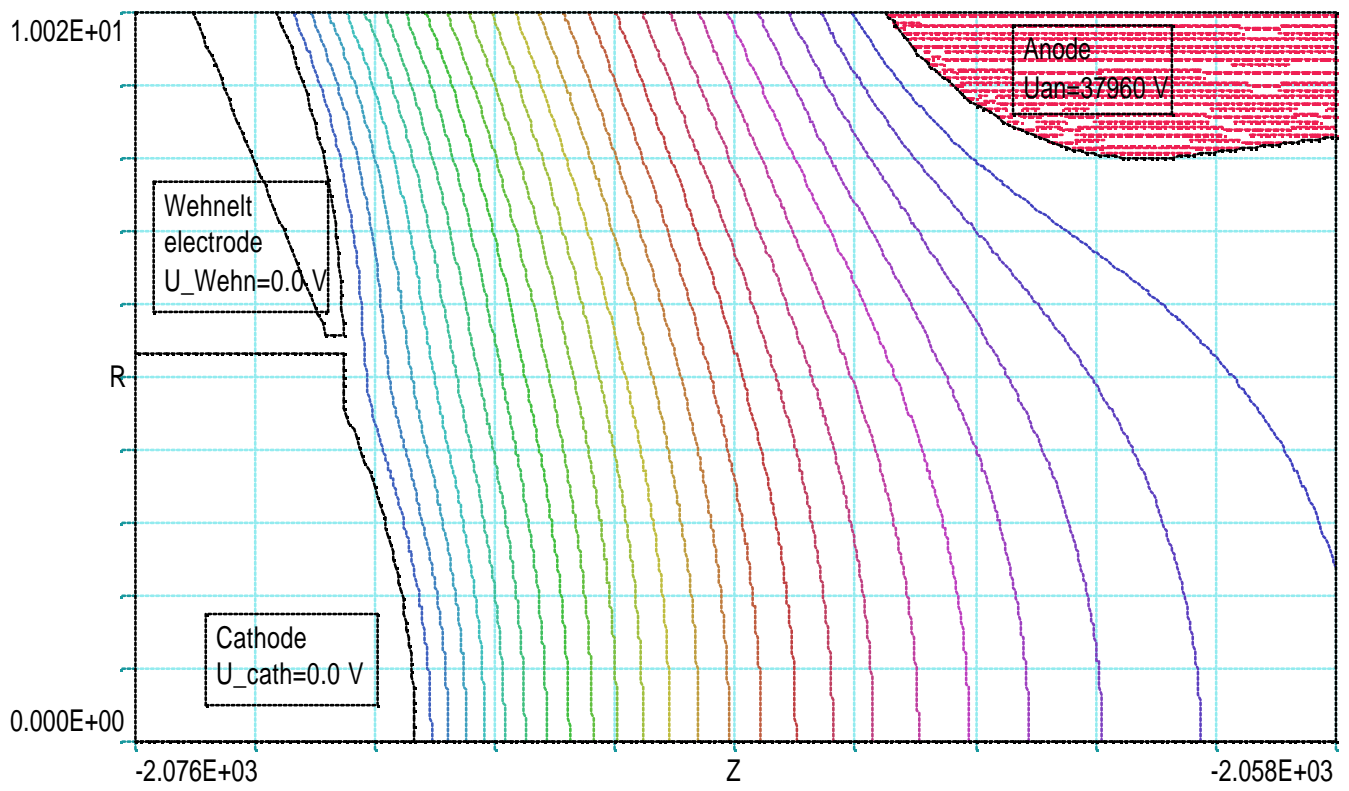

Fig. 5. Electrodes geometry of the high perveance electron gun with not shielded Wehnelt electrode and electric field distribution with zero bias of Wehnelt with respect to the cathode. 


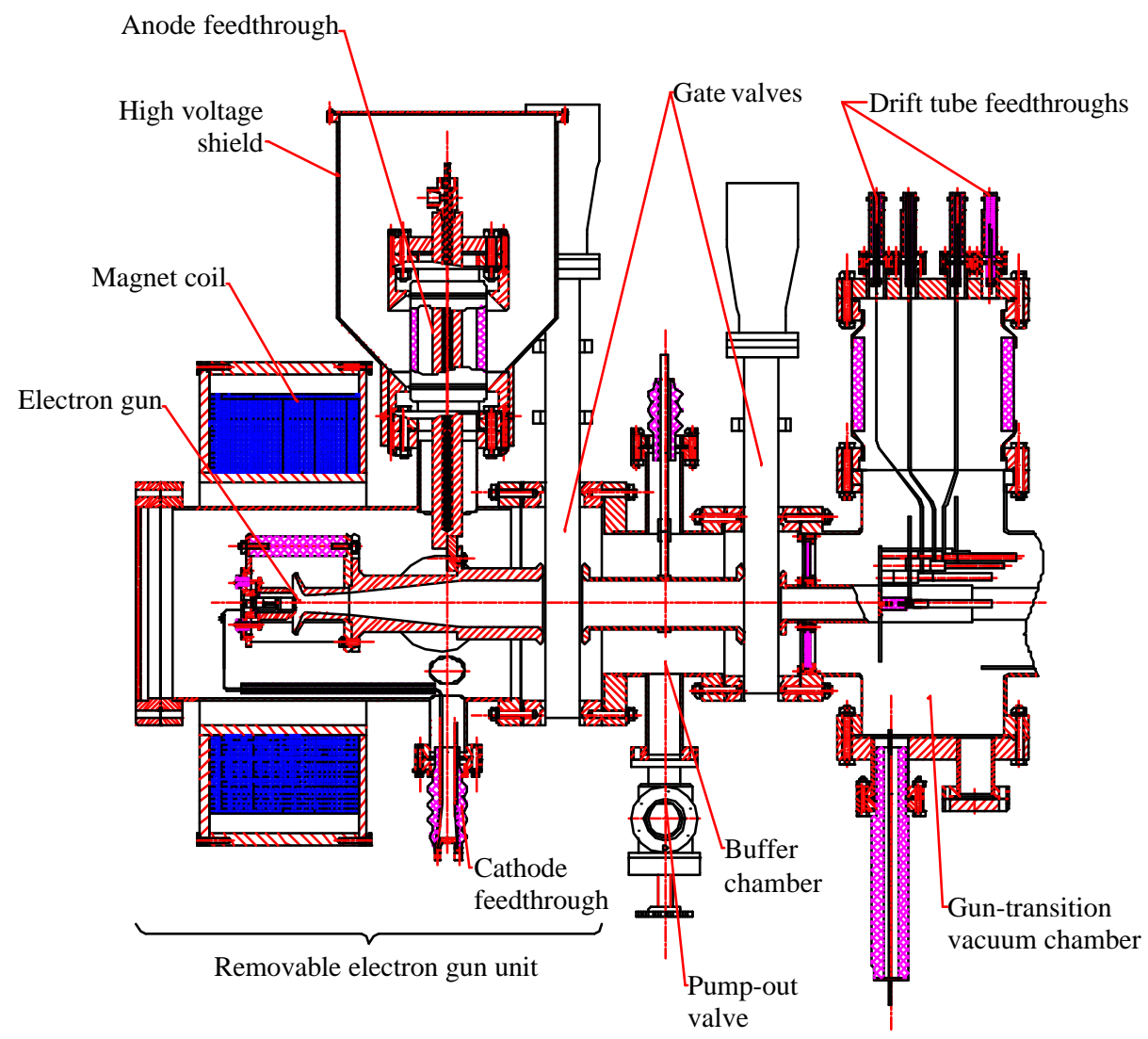

Fig. 6. Removable electron gun assembly with magnet coil.

\section{Simulations and analysis}

The electron gun with several configurations of electrodes has been simulated with electron current in a range of $\mathrm{I}_{\mathrm{el}}=20 \mathrm{~A}$, starting from the cathode to a distance of 200 $\mathrm{mm}$. All simulations have been done with magnetic field on the cathode $0.18 \mathrm{~T}$ using a combined magnetostatic file with Accel main solenoid. The computer simulations have been done with program TRAK [3].

- Low perveance electron gun configuration with not shielded Wehnelt electrode (the geometry of the existing electron gun).

The trajectory plot of the electron beam is presented on Fig. 7. The perveance of this gun is $1.49 \times 10^{-6} \mathrm{~A} / \mathrm{V}^{3 / 2}$ (the same as has the existing gun). One can see that the beam quality is very good, no aberrations can be observed. The radial emission density profile is presented on Fig. 8. This distribution is fairly flat with some increase of emission on the periphery of the cathode.

- High perveance electron gun configuration with not shielded Wehnelt electrode. 
The electron trajectories are presented on Fig. 9 and the emission density profiles are presented on Fig. 10. Even though the perveance of this gun is approximately twice higher than has the gun with prototype geometry the beam quality is very similar to the prototype gun and so is emission density profile. It is possible to control the emission density distribution by changing the potential of Wehnelt electrode, although it requires several kilovolts to obtain a visible change in the emission density distribution.

- High perveance gun configuration with shielded Wehnelt electrode. The trajectories of a 20 A electron beam are presented on Fig. 11. One can see that the peripheral part of the electron beam has aberrations caused by the distorted electric field on the periphery of the cathode. This distortion is produced by the shielded effect of the Wehnelt electrode. This version of the electron gun has also some larger amplitude of radial oscillations than the other versions. The emission current density profiles of this gun with different voltages on the Wehnelt electrode are presented on Fig. 12. The response of emission to the Wehnelt voltage for this Wehnelt geometry is much stronger than for the not shielded geometry. Even with zero bias potential on the Wehnelt electrode with respect to the cathode the emission density on the periphery of the cathode is almost 5 times lower than in the center of the cathode. It is expected that such shaping of the emission profile will result in more uniform distribution of the electron beam power over the electron collector surface than with uniform emission, which is important for the lifetime of the electron collector.

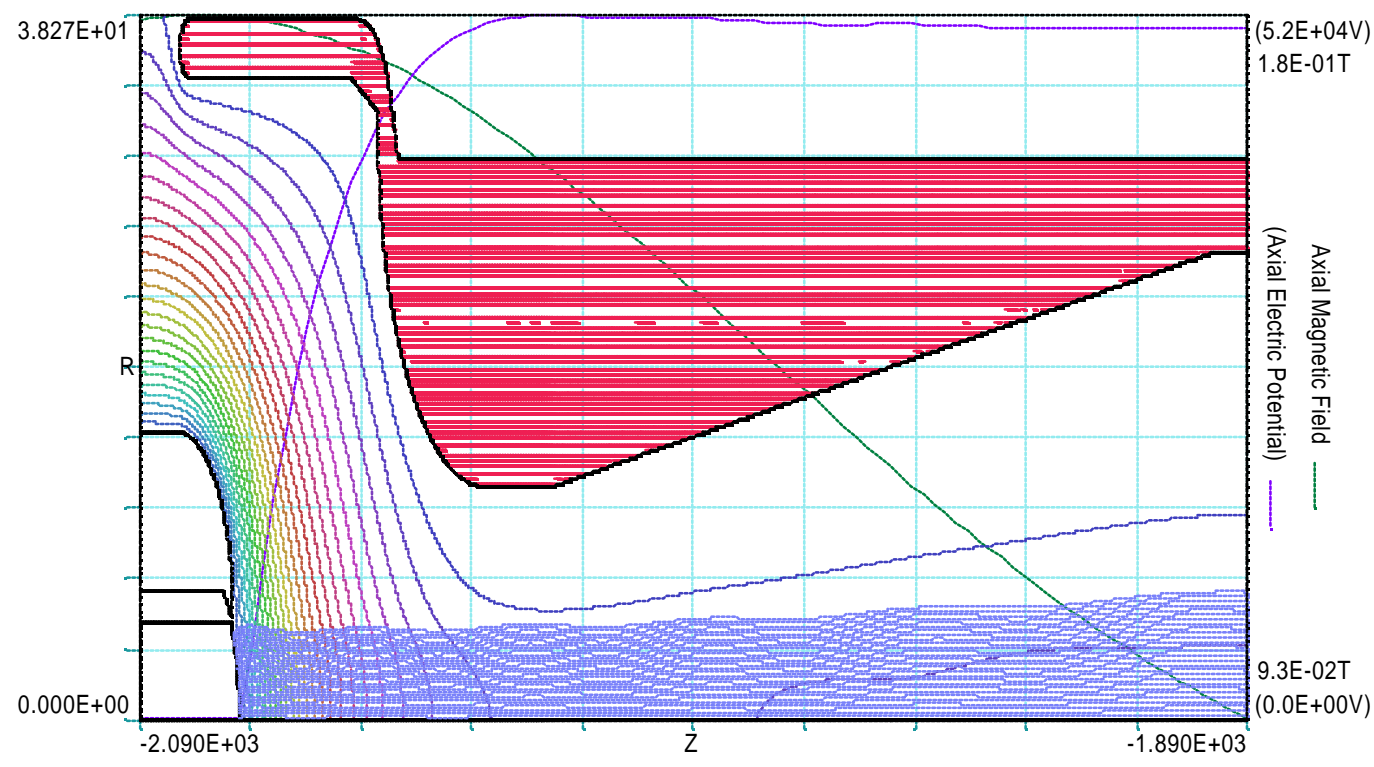

Fig. 7. Simulated electron trajectories with a prototype geometry of the gun electrodes. $\mathrm{I}_{\mathrm{el}}$ $=20 \mathrm{~A}, \mathrm{U}_{\mathrm{an}}=56329 \mathrm{~V}, \mathrm{P}=1.49 \times 10^{-6} \mathrm{~A} / \mathrm{V}^{3 / 2}$. 


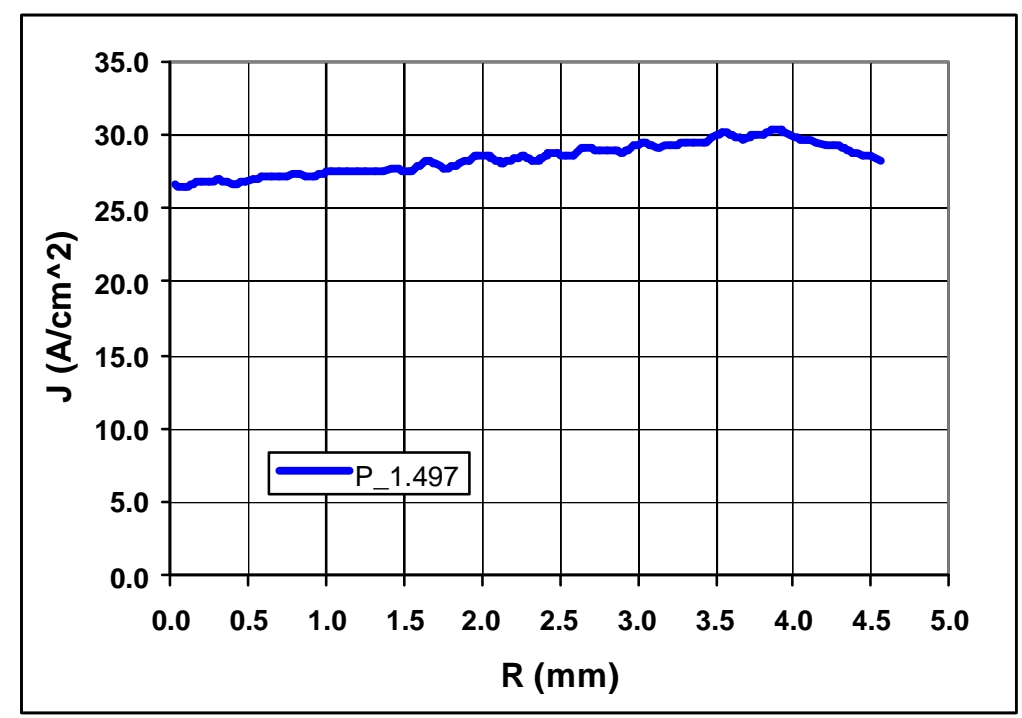

Fig. 8. Radial distribution of the emission current density for prototype geometry of electrodes. $\mathrm{I}_{\mathrm{el}}=20.0 \mathrm{~A}, \mathrm{U}_{\mathrm{an}}=56329 \mathrm{~V}, \mathrm{P}=1.497 \times 10^{-6} \mathrm{~A} / \mathrm{V}^{3 / 2}$.

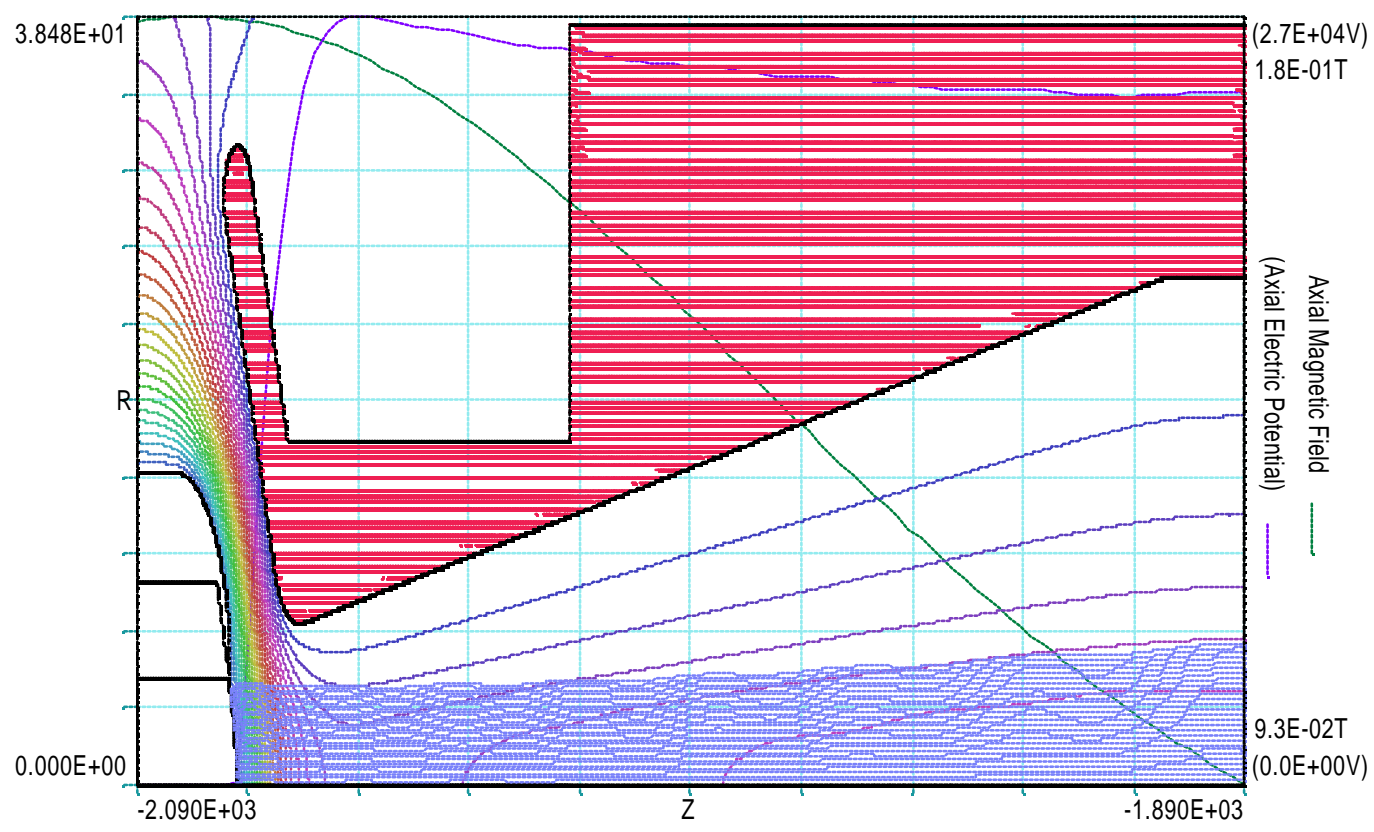

Fig. 9. Simulated electron trajectories for a high perveance version of the gun electrodes with not shielded Wehnelt electrode. $\mathrm{I}_{\mathrm{el}}=20 \mathrm{~A}, \mathrm{U}_{\mathrm{an}}=30968 \mathrm{~V}, \mathrm{P}=3.67 \times 10^{-6} \mathrm{~A} / \mathrm{V}^{3 / 2}$. 


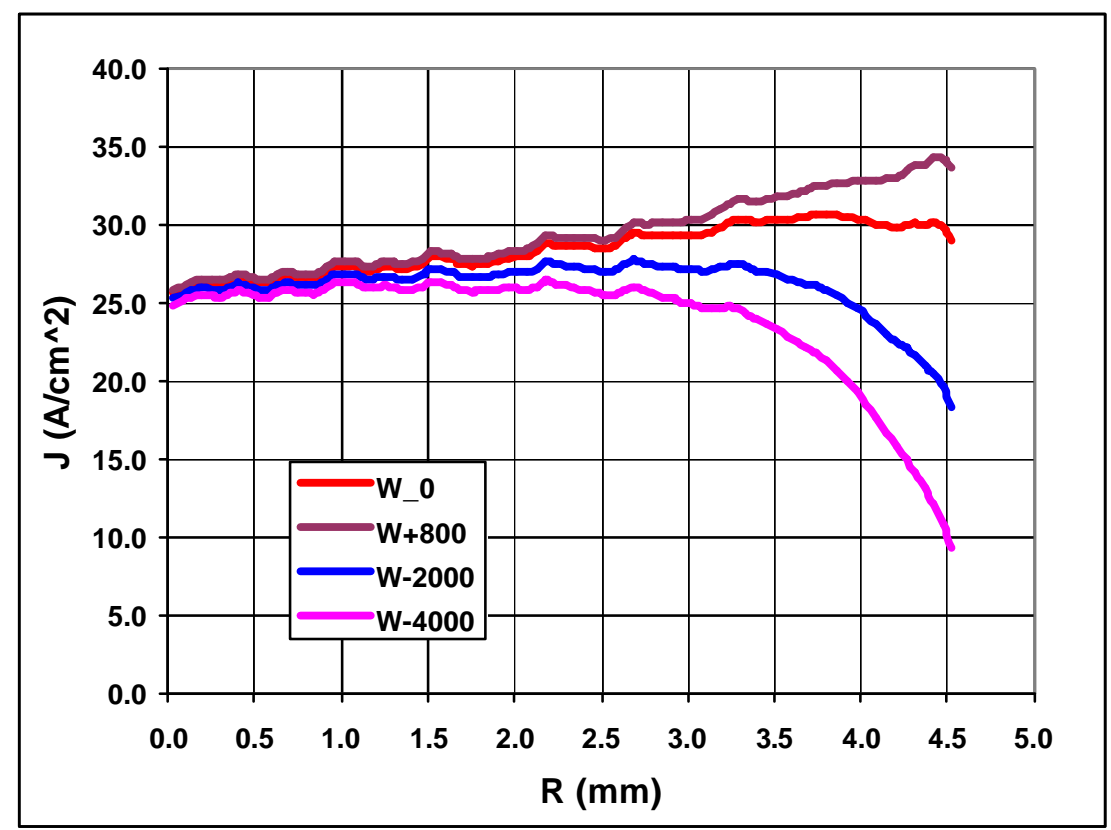

Fig. 10. Radial distribution of the emission current density for a high perveance electron gun with not shielded Wehnelt electrode for a fixed anode voltage $\mathrm{U}_{\mathrm{an}}=30968.0 \mathrm{~V}$ and different voltages on Wehnelt electrode with respect to the cathode $(+800 \mathrm{~V}, 0 \mathrm{~V},-2000 \mathrm{~V}$, and $-4000 \mathrm{~V}$ ). For $\mathrm{U}_{\text {Wehnelt }}=0 \mathrm{~V}$ the electron current $\mathrm{I}_{\mathrm{el}}=20.0 \mathrm{~A}$ and the perveance of the gun $\mathrm{P}=3.67 \times 10^{-6} \mathrm{~A} / \mathrm{V}^{3 / 2}$.

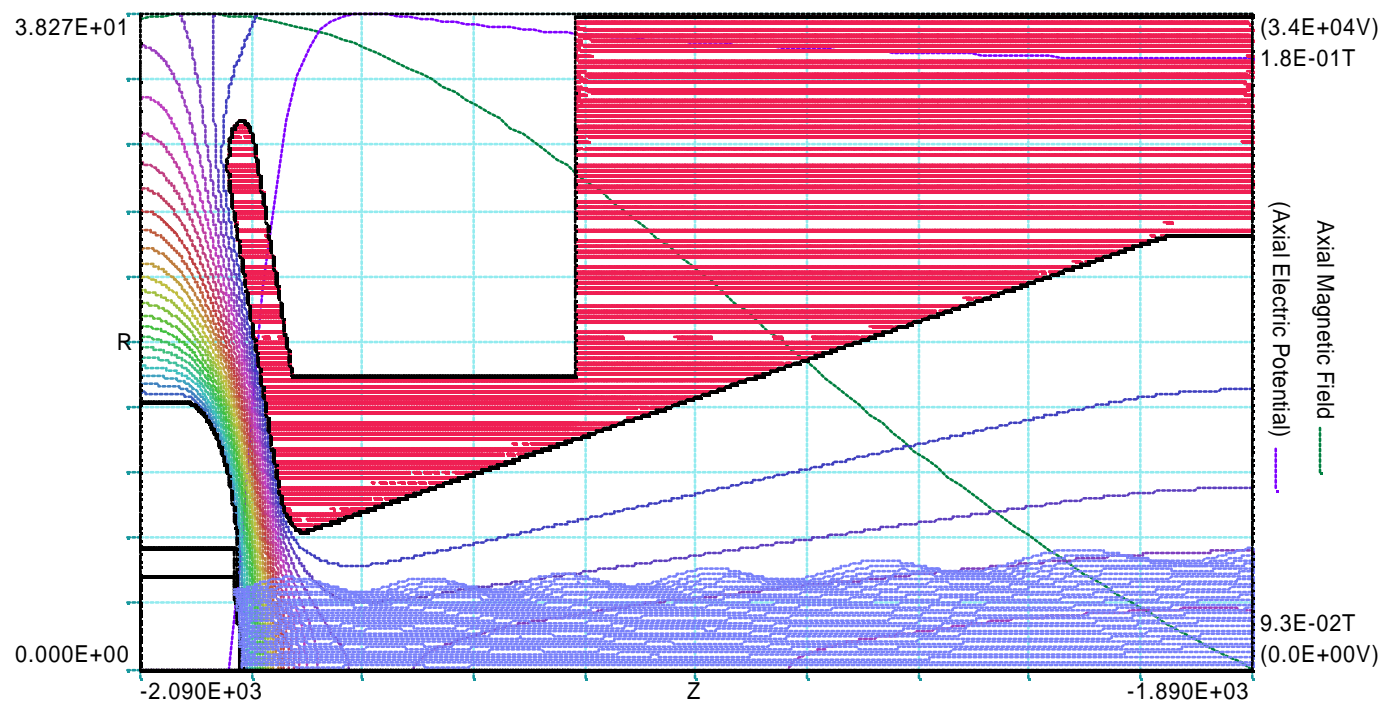

Fig. 11. Simulated electron trajectories for a high perveance version of the gun electrodes with shielded Wehnelt electrode. $\mathrm{I}_{\mathrm{el}}=20 \mathrm{~A}, \mathrm{U}_{\mathrm{an}}=38273 \mathrm{~V}, \mathrm{P}=2.67 \times 10^{-6} \mathrm{~A} / \mathrm{V}^{3 / 2}$. 


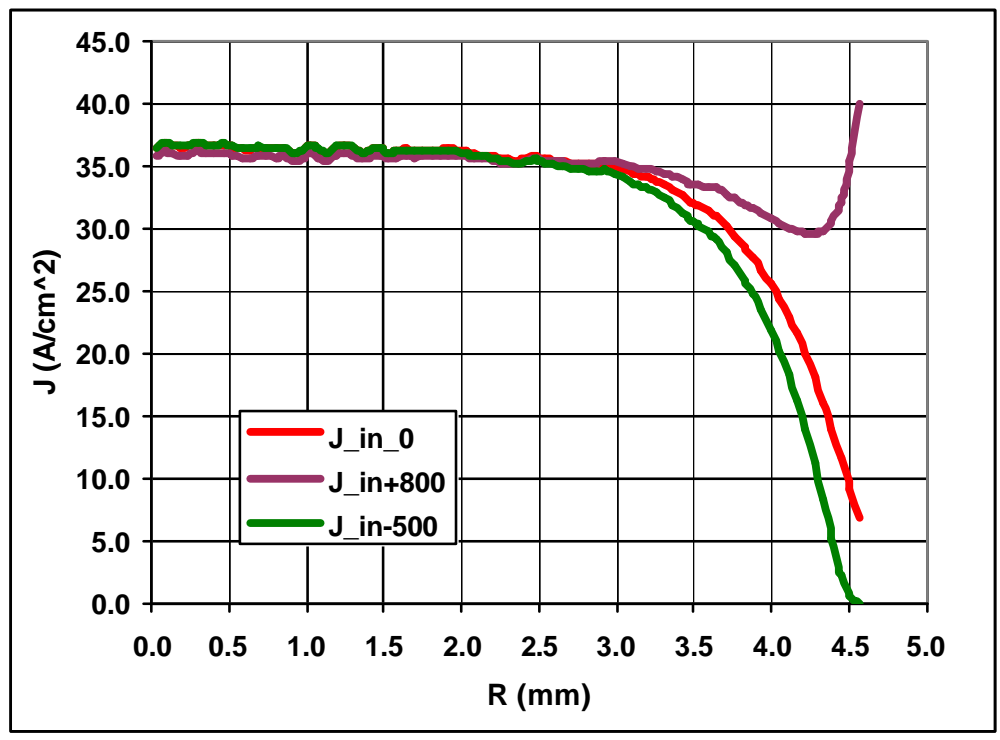

Fig. 12. Radial distribution of the emission current density for a high perveance electron gun with shielded Wehnelt electrode for a fixed anode voltage $U_{a n}=38273.0 \mathrm{~V}$ and different voltages on Wehnelt electrode with respect to the cathode $(+800 \mathrm{~V}, 0 \mathrm{~V}$, and $500 \mathrm{~V}$,$) . For \mathrm{U}_{\mathrm{Wehnelt}}=0 \mathrm{~V}$ the electron current $\mathrm{I}_{\mathrm{el}}=20.0 \mathrm{~A}$ and the perveance of the gun $\mathrm{P}=$ $2,67 \times 10^{-6} \mathrm{~A} / \mathrm{V}^{3 / 2}$.

\section{Cathode}

The cathodes we are planning to use are made of IrCe alloy and are manufactured in Budker Institute of Physics (Novosibirsk, Russia). According to [5] the lifetime of IrCe cathodes with emission current density of $35 \mathrm{~A} / \mathrm{cm}^{2}$ (required for 20 A operation) is approximately 10,000 hours and much longer for emission current density of $17 \mathrm{~A} / \mathrm{cm}^{2}$ (required for 10 A operation). These cathode units have been tested on Test EBIS and demonstrated exceptional performance in operation with electron beam up to $10 \mathrm{~A}$ : they require lower power than $\mathrm{LaB}_{6}$ cathodes and there were no visible deterioration in emission during several hundred hours of operation. The IrCe cathode unit with emission current of $13 \mathrm{~A} / \mathrm{cm}^{2}$ (the same as Test EBIS cathode) operated in Novosibirsk for 40,000 hours [6].

\section{Conclusion}

The electron gun designed for RHIC EBIS is capable of generating the electron beam up to 20 A with parameters sufficient to meet the RHIC EBIS requirements. Low perveance version of the electron gun provides an exceptional beam quality but requires $56 \mathrm{kV}$ anode voltage and has near uniform emission density distribution with slight increase on the periphery. The high perveance version with not shielded Wehnelt electrode generates a 20 A electron beam with almost twice lower voltage than the prototype with similar beam quality and emission density distribution. The high perveance version with shielded geometry trims cathode emission on the periphery 5 times compare to the center and has some lower perveance than with the not shielded Wehnelt. The price for this advantage is peripheral aberrations, increased radial 
oscillations of the electron beam and some higher anode voltage than with not shielded Wehnelt electrode ( $38 \mathrm{kV}$ compare to $31 \mathrm{kV}$ ).

With several options of electrodes configuration we are planning to make a choice based on the experimental study of the performance the gun - electron collector assembly on a bench.

\section{References}

1. A. Kponou, E. Beebe, A. Pikin, G. Kuznetsov, M. Batazova, M. Tiunov, Rev. Sci. Instr., 69, 1998, pp. 1120-1122.

http://scitation.aip.org/getpdf/servlet/GetPDFServlet?filetype=pdf\&id=RSINAK0000690 $\underline{00002001120000001 \& i d t y p e=\text { cvips \&prog }=\text { normal }}$

2. Gennadiy I. Kuznetsov, Marina A. Batazova and Michael A. Tiunov, Proceedings of Electron Beam Ion Sources and Traps and Their Applications: 8th International Symposium, in AIP Conf. Proceedings 572, 2001, pp. 43-47.

3. http://www.fieldp.com/

4. X. Zhang et al., Upgrades of the Tevatron electron lens, Proceedings of 2003 Particle Accelerator Conference, pp. 1781-1783. http://epaper.kek.jp/p03/PAPERS/TPPB077.PDF

5. G. Kuznetsov, High temperature cathodes for high current density, Nuclear Instruments \& Methods in Physics Research, Section A, vol.340, no.1, p. 204-8, 11 Feb. 1994

http://www.sciencedirect.com/science?_ob=MImg\&_imagekey=B6TJM-473FKKT-VB$1 \&$ cdi $=5314 \&$ user $=2422869 \&$ orig $=$ browse \& coverDate $=02 \% 2 \mathrm{~F} 11 \% 2 \mathrm{~F} 1994 \&$ sk $=9$ 96599998\&view=c\&wchp=dGLbVlbzSkWA\&md5=aaedda6851d4517a1f978f44ec1a8c3d\&ie=/sdarticle.pdf

6. G. Kuznetsov, Private communication. 